\title{
Two male study groups with adiposity and hypertriglyceridemia were at risk for hypertension and alcohol use declined renal endothelium
}

\author{
Ruth-Maria Korth
}

Research in General Medicine FiDA, Munich, Germany; fida@,i-dial.de

Received 11 November 2012; revised 12 December 2012; accepted 18 December 2012

\begin{abstract}
Men who attended a Bavarian General Medicine Practice were confidentially invented here. Two male study groups were enrolled to characterize adiposity or hypertriglyceridemia showing that these men were at baseline risk for hypertension [1]. Adverse alcohol consumption mediated dysfunction of renal endothelium as shown here and before [1]. This study found that alcohol use aggravated dyslipidemia, fatty liver disease and critical fasting blood glucose of obese men predicting then late hepatorenal disorders. Overall, two male study groups showed a relevant proportion of men who reported alcohol consumption showing then critical morning urines indicating dysfunction of renal endothelium. The present report looked also at healthy men who reported positive lifestyle behaviour and at men with nonalcohol adiposity and nonalcohol hypertriglyceridemia who then showed normal morning urines indicating functional renal endothelium. Relatively young men at risk were motivated to replace adverse alcohol use by healthy liquids without alcohol and by higher quality of food.
\end{abstract}

Keywords: Adiposity; Hypertriglyceridemia; Hypertension; Alcohol Use; Dysfunctional Endothelium; Fatty Liver Disease

\section{INTRODUCTION}

Men were examined in a Practice of General Medicine and baseline measures were anonymously scored and explored since 1990 [1,2]. A direct relationship between adiposity, hypertriglyceridemia and hypertension is shown with evidence whereby self-reported alcohol use mediates urine pathology testing here albuminuria [1]. Baseline measures of the first male study group provide evi- dence that adiposity or alcohol use are associated with lowering of HDL-cholesterol and submaximal ratio of serum albumin to triglycerides in the absence of type 2 diabetes mellitus or infections ( $\mathrm{C} 1: \mathrm{n}=84,31 \pm 12$ years) [1]. The second male study group integrated fatty liver disease and type 2 diabetes mellitus as men often reported here alcohol use ( $\mathrm{C} 2: \mathrm{n}=60$, aged $37 \pm 10$ years). About half of the male participants were healthy and disowned alcohol use.

The original study program provided knowledge about disturbed plasma compartments which carry alcohol-related ether phospholipids as it is shown that ether phospholipids transform lipoproteins and serum albumin [38]. Alcohol use declines endothelial barriers so that transformed lipoproteins and serum albumin can interact with subendothelial cells [1-8].

Clinical background art shows that urinary albumin predicts stroke, renal failure and cardiovascular disorders [9]. Other prospective studies determine the major arterial risk factors consisting of aging, high cholesterol, smoking, high blood pressure, type 2 diabetes mellitus which predict arterial disorders and stroke [10]. It is known that smoking and/or type 2 diabetes mellitus are major risk factors of obese persons and that obese persons with multiple risk factors are at risk for arterial lesions at 21 to 39 years of age [11]. It is also known that obese persons are at risk for nonalcoholic steatohepatitis implicating healthy food comprising antioxidant tocopheroles, appropriate pharmacotherapy and cessation of smoking $[11,12]$.

Obese women care about health in balance in view of the higher risk of obese children and adolescents

(www.fidabus.com) [13]. For example, local female obesity groups show progress as the majority of obese women recently disowns daily alcohol consumption having then normal morning urines [8].

This report aimed to motivate relatively young men who had to perceive and change risky habits. Indeed, men with adiposity and/or hypertriglyceridemia often reported adverse alcohol use showing hypertension and dysfunction of renal endothelium [1]. Other reports show 
that hypertension predicts renal small vessel disease in general including a higher risk for erectile dysfunction and late cerebrovascular disorders [14-16]. This report found that men with adiposity and/or hypertriglyceridemia were sensitive to alcohol use which declined renal endothelium and aggravated fatty liver disease with critical fasting blood glucose.

\section{OBJECTIVES AND METHODS}

Baseline measures were invented here using medical standard procedures in the same form since 1990 [1]. The local ethical authority approved the study program for epidemiological studies with anonymous and confidentially documented data (BLÄK-No 02088, 07026). Evidence has been provided before using two statistical methods [1]. A second male study group was scored and explored here to resolve putative progress using the original study program [1].

Baseline measures of rather unselected men were further enrolled in the practice $(\mathrm{C} 2: \mathrm{n}=60$, aged $37 \pm 10$ years, 2006-2009). Hepatic problems and type 2 diabetes mellitus were integrated. A further 68 men were treated and initially excluded because they had infections or attended the practice only for counceling, vaccination or drug therapy.

Baseline measures were confidentially enrolled and age, body mass index, blood pressure, morning urines, clinical chemistry and personal history were documented to evaluate blinded data [1]. Blood pressure was examined after 5 - $10 \mathrm{~min}$ of rest and risky persons were monitored in respect to the guidelines (not shown here) [15]. Preprandial blood samples, glucose profiles and morning urines were screened as well. Silent infections were further excluded testing $\mathrm{C}$-reactive proteins (CRP $\leq 0.5$ $\mathrm{mg} / \mathrm{dl})$.

Higher-risk divisions were characterized testing metabolic profiles to characterize men with adiposity $\left(\mathrm{BMI}_{2} \geq\right.$ $29 \mathrm{~kg} / \mathrm{m}^{2}, 11$ out of $\left.60(18 \%)\right)$ or with high triglycerides (Trig: $\geq 160 \mathrm{mg} / \mathrm{dl}, 16$ out of $60(27 \%)$ ). The negative outcome was determined with blood pressure (RR: $\geq$ 135/85 mmHg), critical cholesterol (C: $\geq 200 \mathrm{mg} / \mathrm{dl}$ ), LDL-cholesterol (LDL-C: $\geq 150 \mathrm{mg} / \mathrm{dl}$ ), glucose profiles $(\mathrm{GLC} \geq 100 \mathrm{mg} / \mathrm{dl}, 1 \mathrm{hpp} \geq 140 \mathrm{mg} / \mathrm{dl}, \mathrm{HbA} 1 \mathrm{c} \geq 6 \%)$ and hepatic gamma glutamyl transferase (GGT $\geq 30 \mathrm{U} / \mathrm{l})$. Dyslipidemia was determined here with lowering of HDLcholesterol (HDL-C: $\leq 45 \mathrm{mg} / \mathrm{dl}$ ), elevated cholesterol ratio ( $\mathrm{LDL} / \mathrm{HDL} \geq 4)$. The ratio of serum albumin to triglycerides was also tested $(\mathrm{Alb} /$ Trig $<40)[1]$.

Morning urine samples were tested and means of albuminuria were calculated (urinary albumin $\geq 20 \mathrm{mg} / \mathrm{l}$, Microalbustix, Bayer, Germany). Hematuria and/or proteinuria (without leukocyturia) were initially examined as shown before [1]. In short, urinary protein analysis confirmed albuminuria and excluded pathological proteins whereby urine microscopy further excluded urological or renal disorders at baseline.

\section{RESULTS}

\subsection{Estimated Baseline Measures of the First Male Study Group (C1)}

This original first study group has shown the locally relevant proportion of men with overweight and adiposity $\left(\mathrm{C} 1 / \mathrm{BMI}_{1}: 17\right.$ of $84, \mathrm{C} 1 / \mathrm{BMI}_{2}: 19$ out of $84, \mathrm{BMI}_{1+2}$ : $43 \%$ of 84$)$. Adiposity overlapped with high triglycerides (C1/Trig: 20 out of $84(24 \%)$ ) [1]. Men with adiposity had significantly higher diastolic blood pressure $(\mathrm{p}<$ $0.05)$, lower HDL-cholesterol $(\mathrm{p}<0.05)$ and lower ratio of serum albumin to triglycerides $(\mathrm{p}<0.05)$ compared to men with normal weight [1]. Men with critical body weight often reported alcohol use and these risky men showed significantly higher blood pressure and more often urine pathology compared to men who disowned alcohol problems ( $\mathrm{p}<0.05,19$ of $84(23 \%),>70 \pm 12 \mathrm{~g}$ ethanol/day) [1]. No direct relationship was found between urine pathology and rise of blood pressure [1].

\subsection{Characteristics of the Second Male Study Group (C2)}

Likewise, the second male study group showed a relevant proportion of men who had overweight or obesity $\left(\mathrm{BMI}_{1+2}: 26 \pm 4 \mathrm{~kg} / \mathrm{m}^{2}: 27\right.$ out of $\left.60(45 \%)\right)$. In a broad overview, lipid compartments were in the normal range and cholesterol distribution was normal (see Table 1). However, fasting triglycerides, fasting blood glucose and liver values showed high variance indicating inhomogenous risk divisions which were distinguished hereinafter (HDL-C: $50 \pm 17 \mathrm{mg} / \mathrm{dl}$ with LDL/HDL: $2.9 \pm 1.8$, Alb/Trig: $51 \pm 27$, GGT: $60 \pm 90 \mathrm{U} / 1)$. Indeed, a relevant proportion of men reported heavy alcohol use (22 out of $60(37 \%))$ and half of these alcohol using men showed urine pathology (10 out of $60(17 \%))$ and critical liver values (13 out of $60(22 \%)$ ). Smoking overlapped (see Table 1).

The good message was, that about half of the men attending the General Medicine practice were healthy and disowned alcohol problems (57\% of 60$)$. These men showed normal weight, normal systolic and diastolic blood pressure, normal lipid profiles and normal fasting blood glucose $\left(24 \pm 2 \mathrm{~kg} / \mathrm{m}^{2}\right.$, RR: $124 \pm 13 / 84 \pm 19$ $\mathrm{mmHg}$, GLC: $67 \pm 21$, CRP: $0.4 \pm 01 \mathrm{mg} / \mathrm{dl}, 34$ out of $60)$. Healthy men showed normal liver values (GGT: 17 $\pm 10 \mathrm{U} / \mathrm{l})$ and a normal proportion of microalbuminuria $(7 \%$ of 60$)$. 
Table 1. Characteristics of men and classified metabolic profiles $(C 2: n=60)$. High blood pressure $(\mathrm{RR})$, adverse habits $($ AHA: $>60$ $\mathrm{g}$ ethanol/day), hepatic GGT(U/l), low serum albumin to triglyceride ratio (Alb/Trig $<40)$, urines with albuminuria (mg/l) were found.

\begin{tabular}{|c|c|c|c|c|c|c|c|c|c|c|c|c|c|c|}
\hline Outcome: & Age & Fast.Gluc. & Chol. & Trig. & LDL-C & HDL-C & BMI & AHA & Cig & Syst.RR & Diast.RR & Urines & Alb/Trig & GGT \\
\hline \multicolumn{15}{|c|}{ 1) Characteristics of the second male study group (C2): } \\
\hline $\mathrm{n}=60$ & $37 \pm 10$ & $91 \pm 15$ & $202 \pm 51$ & $144 \pm 125$ & $134 \pm 44$ & $50 \pm 17$ & $26 \pm 4$ & $\mathrm{n}=21$ & $\mathrm{n}=20$ & $129 \pm 15$ & $88 \pm 10$ & $\mathrm{n}=15$ & $51 \pm 27$ & $60 \pm 90$ \\
\hline$\%$ of $60:$ & years & $\mathrm{mg} / \mathrm{dl}$ & $\mathrm{mg} / \mathrm{dl}$ & $\mathrm{mg} / \mathrm{dl}$ & $\mathrm{mg} / \mathrm{dl}$ & $\mathrm{mg} / \mathrm{dl}$ & $\mathrm{kg} / \mathrm{m}^{2}$ & $35 \%$ & $33 \%$ & $\mathrm{mmHg}$ & $\mathrm{mmHg}$ & $25 \%$ & & $\mathrm{U} / 1$ \\
\hline \multicolumn{15}{|c|}{ 2) Adiposity (C2/BMI2 $\geq 29 \mathrm{~kg} / \mathrm{m} 2,11$ out of $60(18 \%)$ ): } \\
\hline 11 of 60 & $42 \pm 9$ & $97 \pm 18$ & $245 \pm 51$ & $286 \pm 214$ & $158 \pm 48$ & $40 \pm 14$ & $33 \pm 4$ & $\mathrm{n}=7$ & $\mathrm{n}=5$ & $138 \pm 16$ & $96 \pm 11$ & $\mathrm{n}=5$ & $24 \pm 14$ & $188 \pm 148$ \\
\hline Albuminuri & ia, $\%$ of & & & & & & & & & & & $45 \%$ & & \\
\hline \multicolumn{15}{|c|}{ Nonalcohol adiposity (C2/BMI2, 4 of $60(7 \%))$ : } \\
\hline $36 \%$ of 11 & $43 \pm 12$ & $104 \pm 15$ & $195 \pm 26$ & $117 \pm 46$ & $142 \pm 23$ & $57 \pm 28$ & $36 \pm 8$ & non & $\mathrm{n}=1$ & $138 \pm 18$ & $102 \pm 9$ & $\mathrm{n}=1$ & $50 \pm 4$ & $65 \pm 3$ \\
\hline \multicolumn{15}{|c|}{ 3) Hypertriglyceridemia (C2/Trig $\geq 160 \mathrm{mg} / \mathrm{dl}, 16$ of $60(27 \%))$ : } \\
\hline 16 of 60 & $42 \pm 10$ & $99 \pm 13$ & $254 \pm 50$ & $276 \pm 152$ & $171 \pm 45$ & $47 \pm 14$ & $29 \pm 5$ & $\mathrm{n}=11$ & $\mathrm{n}=10$ & $129 \pm 19$ & $92 \pm 15$ & $\mathrm{n}=9$ & $19 \pm 6$ & $127 \pm 129$ \\
\hline Albuminuri & ia $\%$ of 1 & & & & & & & & & & & $56 \%$ & & \\
\hline \multicolumn{15}{|c|}{ Nonalcohol Hypertriglyceridemia (C2/Trig: 5 out of $60(8 \%))$ : } \\
\hline $31 \%$ of $16:$ & $41 \pm 14$ & $95 \pm 10$ & $216 \pm 51$ & $224 \pm 44$ & $158 \pm 21$ & $48 \pm 15$ & $26 \pm 7$ & non & $\mathrm{n}=1$ & $125 \pm 13$ & $90 \pm 22$ & $\mathrm{n}=1$ & $28 \pm 6$ & $58 \pm 16$ \\
\hline
\end{tabular}

\subsection{Adiposity of the Second Male Study Group (C2)}

Next, men with adiposity were characterized $\left(\mathrm{BMI}_{2}\right.$; $33 \pm 4 \mathrm{~kg} / \mathrm{m}^{2}$; see Table 1). Obese men showed high fasting triglycerides, diastolic hypertension and tended to critical fasting blood glucose (see Table 1). Dyslipidemia was found with high LDL-cholesterol, low HDL-cholesterol, elevated LDL/HDL and submaximal Alb/Trig (LDL-C: $158 \pm 48$, HDL-C: $40 \pm 14 \mathrm{mg} / \mathrm{dl}$, LDL/HDL: $5.5 \pm 1.8$, Alb/Trig: $24 \pm 14$ ). These obese dyslipidemic men tended to have critical fasting blood glucose at baseline (GLC: $97 \pm 18 \mathrm{mg} / \mathrm{dl}$, CRP $0.6 \pm 0.3 \mathrm{mg} / \mathrm{dl}$ ).

About half of the men in the obesity group reported adverse alcohol consumption showing then urine patho$\operatorname{logy}(24 \pm 14 \mathrm{mg} / \mathrm{l}$ urinary albumin, see Table 1). Critical liver values were aggravated when obese men reported alcohol use (AHA-GGT: $188 \pm 148$ vs NA-BMI $2: 65$ $\pm 3 \mathrm{U} / \mathrm{l})$. Obese men who disowned alcohol consumption also showed elevated diastolic blood pressure and critical fasting blood glucose (see Table 1).

This report looked carefully at this small group characterized with nonalcohol adiposity. Obese men who disowned alcohol use had normal HDL-cholesterol, normal cholesterol ratio and normal Alb/Trig (HDL-C: $57 \pm 28$ $\mathrm{mg} / \mathrm{dl}$ with LDL/HDL: $3.3 \pm 0.4$, Alb/Trig $50 \pm 14$ ). Overall, normal morning urines were found among obese men who disowned alcohol use (see Table 1). Only one abstinent obese men showed microalbuminuria who suffered of type 2 diabetes mellitus.

However, the number of obese men who disowned daily alcohol consumption was small and these men with nonalcohol adiposity showed diastolic hypertension as well (NA-BMI $2: 34 \pm 4 \mathrm{~kg} / \mathrm{m}^{2}, 133 \pm 18 / 96 \pm 11 \mathrm{mmHg}$, $23 \%$ of 30 out of $\mathrm{C} 1+\mathrm{C} 2$ ). Again, normal morning urines were found indicating functional renal endothelium.

Altogether, adiposity, hypertriglyceridemia and alcohol use overlapped and no relevant difference was found between both study groups. Estimated subgroups consisting of obese participants showed higher diastolic blood pressure compared to participants with normal weight ( $p$ $<0.05$ ). However, the small number of obese men who disowned alcohol use limited here a direct comparison between metabolic profiles of men with alcohol-related versus nonalcohol adiposity.

Indeed, obese men were at direct risk for diastolic hypertension and often reported adverse alcohol consumption which declined renal endothelium, triggered dyslipidemia and aggravated fatty liver disease. The good message was that obese men who reported healthy lifestyle showed normal HDL-cholesterol and functional endothelium.

\subsection{Hypertriglyceridemia of the Second Male Study Group (C2)}

Hypertriglyceridemia was characterized with baseline measures and these men were obese and showed diastolic hypertension and critical fasting blood glucose (see Table 1). In addition, men with hypertriglyceridemia showed high cholesterol ratio whereby HDL-cholesterol was still normal (LDL-C: $171 \pm 45$, HDL-C: $47 \pm 14$ $\mathrm{mg} / \mathrm{dl}$ with LDL/HDL: $4.0 \pm 2.4$, Alb/Trig $19 \pm 6$, CRP $0.6 \pm 0.3 \mathrm{mg} / \mathrm{dl}$ ). The triglyceride-related baseline markers varied indicating inhomogenous subgroups which were distinguished hereinafter. 
Four diabetic men were found in this hypertriglyceridemia division (GLC: $109 \pm 6 \mathrm{mg} / \mathrm{dl}, 1 \mathrm{~h} \mathrm{pp:} 222 \pm 54,2$ h pp: $126 \pm 41$, HbAlc: $6.5 \% \pm 1.3 \%$, 4 out of $60(7 \%))$. These men with type 2 diabetes mellitus suffered of hypertriglyceridemia and only one of these four diabetic men disowned drinking and smoking and showed diabetic microalbuminuria as well.

In addition, men with high triglycerides often reported heavy alcohol consumption and these men showed urinary albumin indicating dysfunctional renal endothelium (44 $\pm 31 \mathrm{mg}$ urinary albumin/1, see Table 1). Self-reported alcohol use of hypertriglyceridemic men aggravated fatty liver disease compared to nonalcohol hypertriglyc0 eridemia (AHA-GGT: $127 \pm 129$ vs NA-Trig: $58 \pm 16$ $\mathrm{U} / 1)$.

This report looked carefully at the small group of men with nonalcohol hypertriglyceridemia showing critical diastolic blood pressure as well (see Table 1). The good message was that nonalcohol hypertriglyceridemia showed normal HDL-cholesterol despite critical level of LDLcholesterol and moderate triglyceride ratio (LDL-C: 158 \pm 21 , HDL-C: $48 \pm 15 \mathrm{mg} / \mathrm{dl}$ with LDL/HDL: $3.4 \pm 0.4$, Alb/Trig: $28 \pm 6$ ). Overall, nonalcohol hypertriglyceridemia was paralleled with normal morning urines indicating functional renal endothelium (see Table 1).

However, the number of abstinent hypertriglyceridemic men was small (NA-Trig: $205 \pm 27 \mathrm{mg} / \mathrm{dl}, 22 \%$ of 36 out of $\mathrm{C} 1+\mathrm{C} 2$ ) and diastolic hypertension was observed again with nonalcohol hypertriglyceridemia (133 $\pm 13 / 92$ $\pm 10 \mathrm{mmHg}$ ). Overall, morning urines were normal indicating functional renal endothelium.

Altogether, hypertriglyceridemia, obesity and alcohol overlapped and no relevant differences were found between both study groups. Estimated subgroups consisting of hypertriglyceridemic participants showed higher diastolic blood pressure compared to participants with normal triglycerides $(p<0.05)$. However, the small number of men with nonalcohol hypertriglyceridemia limited here a direct comparison between metabolic profiles of men with alcohol-related versus nonalcohol hypertriglyceridemia.

Indeed, men with hypertriglyceridemia tended to be obese and were at direct risk for diastolic hypertension whereby alcohol use declined renal endothelium and aggravated fatty liver disease which was paralleled with type 2 diabetes mellitus. The good message was that men with nonalcohol hypertriglyceridemia showed functional renal endothelium.

\section{DISCUSSION}

Men with adiposity and/or high triglycerides were at direct risk for diastolic hypertension. About half of the men who attended the medical practice were healthy and reported a positive lifestyle behaviour. These healthy men had normal blood pressure, normal metabolic baseline measures, functional endothelium and normal ratio of serum albumin to triglycerides (Alb/Trig). The locally relevant problem was that men often reported adverse alcohol consumption which declined renal endothelium and aggravated dyslipidemia, fatty liver disease and type 2 diabetes mellitus. Adiposity, hypertriglyceridemia and alcohol use overlapped and these risky men showed diastolic hypertension, critical liver values, dyslipidemia and critical fasting blood glucose. Evidence has been provided before that adverse alcohol consumption declines endothelium and also that hypertriglyceridemia mediates diastolic hypertension whereby evidence is provided for the first time with the original study group [1].

The locally relevant study program is initially based on original science showing that human endothelial cells express alkyl receptors and also lipases, namely phospholipases and acetylhydrolases on outer membranes which form and release upregulatory lysophospholipids and fatty acids activating then perivascular cells [17]. Alcohol use declines endothelial barriers so that transformed lipoproteins and/or serum albumin can activate subendothelial cells whereby shown urinary albumin predicts small vessel disease [1-9]. Women with non-alcohol adiposity and/ or hypertriglyceridemia are also at risk for hypertension $[1,8]$. Likewise, mutants of acetylhydrolases predict stroke as shown with Japanese persons [18]. Moreover, alcohol use, hyperlipidemia and adiposity are correlated with lowering of HDL-cholesterol and with low ratio of serum albumin to triglycerides (Alb/Trig < 40) [1,2].

As known, hyperlipidemia activates endothelium and also endothelial lipases which might explain at least in part hypertension in the presence of nonalcohol hypertriglyceridemia which is shown before with abstinent obese women [8]. Background art discloses mutations of the lipase gene family including endothelial lipases whereby lipases trigger hypertriglyceridemia and/or metabolic syndromes also on the nuclear level [19]. Other genetic variants encode hepatic triacylglycerol lipases and acyltransferases (PNLP3) which are related with nonalcoholic fatty liver disease whereby adiposity is a known additive risk factor [20].

The classical antihypertensive strategies recommend cessation of smoking and reduced uptake of sodium and saturated fat to propose low fat milk products and fresh vegetables [21]. Reduced energy uptake is recommended to replace adverse carbohydrates by healthy food and physical exercising which could help obese persons in general [22]. Present guidelines recommend lipid-lowering dietary strategies at baseline considering appropriate pharmacotherapy when obese and/or hyperlipidemic persons cannot solve multiple problems [23]. The Official German guidelines address alcohol problems and 
correctly recommend at least abstinent periods to perceive and change ambivalent denying of addictive alcohol problems [24]. Local training programs begin here with medical examination, self-reported lifestyle problems, abstinent periods, self-testing of blood pressure and morning urines to adapt dietary strategies to medical use integrating also families who suffer of alcohol-related problems [1].

The good message was that healthy men and men with nonalcohol adiposity and/or nonalcohol hypertriglyceridemia showed normal HDL-cholesterol and functional endothelium indicating a healthy defense system. Moreover, oberservational studies show recovery of renal endothelium in a 5 year follow-up of abstinent former alcohol abusers who obtain healthy food and antihypertensive pharmacotherapy [7]. Thus, motivation is provided to perceive and change critical alcohol consumption as relatively young men were at risk for dysfunctional endothelium and related problems.

The clear message was that middle-aged men who suffered of adiposity and/or hypertriglyceridemia often reported alcohol use and/or smoking and half of these men showed dysfunctional renal endothelium predicting endothelium-related gender problems implicating to cede drinking and smoking $[1,11]$. Thus, obese and/or hypertriglyceridemic men who reported alcohol use perceived herewith that they placed themselves into a direct risk class for hypertension, dysfunctional endothelium, dyslipidemia, steatohepatitis and type 2 diabetes mellitus. Indeed, alcohol use aggravated the injurious outcome and triggered a cluster of adverse symptoms keeping in mind that obese men were at risk for hypertension and fatty liver disease at baseline. Background art discloses that hypertension is a major risk factor for small vessel disease, cerebrovascular disorders and/or erectile dysfunction [9,1416].

Telemedical counseling monitored individuals who suffered of adiposity and who wanted to replace unhealthy food and alcohol use. Medical counseling of obese persons is not easy regarding the high discipline which is needed to change risky habits. This report aimed to motivate change of alcohol use showing the additive harm and negative outcome of alcohol-related hypertriglyceridemia. Physicians consider lipid lowering and/or antihypertensive pharmacotherapy when obese and/or hyperlipidemic persons cannot change multiple problems considering the guidelines [23]. This study showed that hypertriglyceridemia is an independent risk factor for diastolic hypertension and triggers multiple risk factors of obese persons, namely dyslipidemia, fatty liver disease and type 2 diabetes mellitus. Risky men showed unchanged risk profiles while obese women recently tend to disown alcohol use having then normal morning urines [8].

The good message was that about half of the men at- tending a General Medicine practice reported healthy lifestyle and showed normal biomarkers. Men with nonalcohol adiposity and/or nonalcohol hypertriglyceridemia also showed normal HDL-cholesterol and functional renal endothelium indicating at least a healthy defense system in the presence of diastolic hypertension. Dietary strategies were adapted here to support the endogenous defense system replacing alcohol use, sodium, saturated fat and adverse carbohydrates by healthy liquids without alcohol adapting then healthy food for individual use (www.fidabus.com, www.fida-aha.com) [25,26]. Medical monitoring aimed to skill self-testing of blood pressure and morning urines to change adverse habits and to improve the quality of food.

\section{CONCLUSION}

Middle-aged men with adiposity and/or hypertriglyceridemia were correlated with diastolic hypertension at baseline. These men attending a Bavarian practice of General Medicine showed unchanged higher-risk profiles whereby alcohol use aggravated dyslipidemia, fatty liver disease and glucose profiles. Benefit was shown with obese and/or hypertriglyceridemic men who disowned daily alcohol consumption because normal morning urines indicated functional renal endothelium. Another good message was that obese and/or hypertriglyceridemic men who disowned alcohol use had normal HDL-cholesterol indicating a healthy defense system in the presence of diastolic hypertension. This report improved risk perception to replace adverse alcohol use by healthy liquids and higher quality of food. Self management was skilled with testing of morning urines and blood pressure.

\section{REFERENCES}

[1] Korth, R.M. (2006) Gender obesity, alcohol use, hyperlipidemia, hypertension and decline of renal endothelial barriers. Journal of Men's Health and Gender, 3, 279-289. doi:10.1016/j.jmhg.2005.08.006

[2] Korth, R.M. (2002) AHA-syndromes. Chemistry Physics of Lipids, 118, 96-97.

[3] Korth, R., Zimmermann, K. and Richter, W. (1994) Lipoprotein-associated paf (LA-paf) was found in washed human platelets and monocyte-macrophage-like U937 cells. Chemistry Physic of Lipids, 70, 109-119. doi:10.1016/0009-3084(94)90079-5

[4] Korth, R.M. (2000) Comparison of phosphocholines in human plasma and cerebrospinal fluid (CSF). The Faseb Journal, 14, A72.

[5] Korth, R., Bidault, J., Palmatier, R., Beneveniste J. and Ninio, E. (1993) Human platelets release a paf-acether: Acetylhydrolase similar to that in plasma. Lipids, 28, 193-199. doi:10.1007/BF02536639

[6] Korth, R.M. (1997) VLDL and PAF binding to human en- 
dothelial cells. Chemistry and Physics of Lipids, 88, 134.

[7] Korth, R.M. (2005) Gender dyslipidemia and ether phospholipids. The Faseb Journal, 19, 109.

[8] Korth, R.M. (2007) Obesity mediated hypertension while alcohol use declined renal endothelial barriers of women. The Faseb Journal, 21, A1361.

[9] Barzilay, J.J., Peterson, D., Cushman, M., Heckbert, S.R., Cao, J.J., Blaum, C., Tracy, R.P., Klein, R. and Herrington, D.M. (2004) The relationship of cardiovascular risk factors to microalbuminuria in older adults with or without diabetes mellitus or hypertension: The cardiovascular health study. American Kidney Disease, 44, 25-34. doi:10.1053/i.ajkd.2004.03.022

[10] Vasan, R.S. (2005) Relative importance of borderline and elevated levels of coronary heart disease risk factors. Annals of Internal Medicine, 142, 393-402.

[11] Berenson, G.S., Srinivasan, S.R., Bao, W., Newmann, W.P., Tracy, R.E. and Wattigney, W.A. (1998) Association between multiple cardiovascular risk factors and atherosclerosis in children and young adults. New England Journal of Medicine, 338, 1650-1656. doi:10.1056/NEJM199806043382302

[12] Sanyal, A.J., Chalasani, N., Kowdley, K.V., McCullough, A., Diel, A.M., Bass, N.M., Neuschwander-Tetri, B.A., Lavine, J.E., Tonascia, J., Unalp, A., Van Natta, M., Clark, J., Brunt, E.M., Kleiner, D.E., Hoofnagle, J.H. and Robuck, P.R. (2010) Pioglitazone, Vitamin E, or placebo for nonalcoholic steatohepatitis. New Engand Journal of Medicine, 362, 1675-1685. doi:10.1056/NEJMoa0907929

[13] Coustan, D.R., Lowe, L.P., Metzger, B.E. and Dyer, A.R. (2007) The hyperglycemia and adverse pregnany outcome (paving the way for new diagnostic criteria for gestational diabetes mellitus). American Journal Obstetic and Gynecology, 202, 654-657.

[14] Gupta, B.P., Murad, M.H., Clifton, M.M., Prokop, L., Nehra, A. and Kopecky, S.L. (2011) The effect of lifestyle modification and cardiovascular risk factor reduction on erectil dysfunction: A systemic review and metaanalyis. Archives Internal Medicine, 171, 1797-17803. doi:10.1001/archinternmed.2011.440

[15] Bertoiy, M.L., Waring, M.E., Gupta, P.S., Roberts, M.B. and Eaton, C.B. (2011) Hypertension Guidelines Implications. Hypertension, 58, 361-366. doi:10.1161/HYPERTENSIONAHA.111.175463

[16] Querzfurth, H.W. (2010) Mechanism of disease Alzheimer' disease. New England Journal Medicine, 362, 329344.
[17] Korth, R.M., Hirafuji, M., Beneveniste, J. and RussoMarie, F. (1995) Human umbilical vein endothelial cells: Specific binding of platelet-activating factor and cytosolic calcium flux. Biochemical Pharmacology, 49, 1793-1799. doi:10.1016/0006-2952(95)00025-U

[18] Hiramoto, M., Yoshida, H., Imaizumi, T., Yoshimizu, N. and Satoh, K. (1997) A mutation in plasma platetet activating factor acetylhydrolases (VAL279-Phe) is a genetic risk factor for stroke. Stroke, 28, 2417-2420. doi:10.1161/01.STR.28.12.2417

[19] Wen, X.Y., Hegele, R.A., Wang, J., Wang, D.Y., Cheung, J., Wilson, M., Yanyapour, M., Bai, Y., Zhuang, L., Skaug, J., Young, T.K., Conelly, P.W., Koop, B.F., Tsui, L.C. and Stewart, A.K. (2003) Identification of a novel lipase gene mutated in lpd mice wth hypertriglyceridemia and associated with dyslipidemia in humans. Human Molecular Genetics, 12, 1131-1143. doi:10.1093/hmg/ddg124

[20] Romeo, S., Kozlitina, J., Xing, C., Pertsemlidis, A., Cox, D., Pennachio, L.A., Boerwinkle, E., Cohen, J.C. and Hobbs, H. (2008) Genetic variation in PNPLA3 confess susceptibility to nonalcoholic fatty liver disease. Nature Genetics, 40, 1461-1465. doi:10.1038/ng.257

[21] Sacks, F.M., Svetkey, L.P., Vollmer, W.M., Appel, L.J., Bray, G.A., Harsha, D., Obarzanek, E., Conlin, P.R., Miller, E.R., Simons-Morton, D.G., Karanja, N. and Lin, P.H.L.N. and DASH-Sodium Collaboration Research Group (2001) Effects on blood pressure of reduced dietary sodium and the dietary approaches to stop hypertension (DASH) diet. New England Journal Medicine, 344, 3-10. doi:10.1056/NEJM200101043440101

[22] Volek, J.S., Fernandez, M.L., Feinman, R.D. and Phinney, S.D. (2008) Dietary carbohydrate restriction induces a unique metablic state positively affecting atherogenic dyslipidemia, fatty acid partioning, and metabolic syndrome. Progress in Lipid Research, 47, 307-318. doi:10.1016/j.plipres.2008.02.003

[23] Humphries, S. (2011) Task force for the management of dyslipidemias of the European Society of Cardiology and the European Atherosclerosis Society. Atherosclerosis, 21751.

[24] Kraus, A.R. (2008) Alkoholkonsum, alkoholbezogene probleme und trends. Ergebnisse des epidemiologischen suchtsurvey 2003. Robert Koch Institut, Gesundheitsberichterstattung des Bundes, 40.

[25] Korth, R.M. (2000) www.fidabus.com

[26] Korth, R.M. (2001) www.fida-aha.com 